\title{
ADAPTIVE FRAMEWORK FOR UNCERTAINTY ANALYSIS IN ELECTROMAGNETIC FIELD MEASUREMENTS
}

Javier Prieto*, Alonso A. Alonso, Ramón de la Rosa and Albano Carrera LEB - Laboratory of Electronics and Bioengineering, Department of Signal Theory and Communications and Telematic Engineering, Universidad de Valladolid, Paseo de Belén 15, 47011, Valladolid, Spain

*Corresponding author: javier.prieto@uva.es

Received 11 April 2014; revised 16 July 2014; accepted 18 July 2014

Misinterpretation of uncertainty in the measurement of the electromagnetic field (EMF) strength may lead to an underestimation of exposure risk or an overestimation of required measurements. The Guide to the Expression of Uncertainty in Measurement (GUM) has internationally been adopted as a de facto standard for uncertainty assessment. However, analyses under such an approach commonly assume unrealistic static models or neglect relevant prior information, resulting in non-robust uncertainties. This study proposes a principled and systematic framework for uncertainty analysis that fuses information from current measurements and prior knowledge. Such a framework dynamically adapts to data by exploiting a likelihood function based on kernel mixtures and incorporates flexible choices of prior information by applying importance sampling. The validity of the proposed techniques is assessed from measurements performed with a broadband radiation meter and an isotropic field probe. The developed framework significantly outperforms GUM approach, achieving a reduction of $28 \%$ in measurement uncertainty.

\section{INTRODUCTION}

In recent years, some people and organisations have become more concerned about the effects of exposure to electric and magnetic fields (EMFs) on human health from both power and radiofrequency sources ${ }^{(1,2)}$. Power EMFs are generated, for example, in the production, transport, distribution and use of electricity ${ }^{(3,4)}$. Radiofrequency EMFs are produced, for example, by microwave ovens, cordless phones, broadcasting transmissions or medical applications ${ }^{(5,6)}$. As a consequence, official governments and international organisations have unevenly developed optional recommendations and binding policies that establish maximum levels of exposure to EMFs.

In Europe, Recommendation 1999/519/EC ${ }^{(7)}$ and Directive $2013 / 35 / \mathrm{EU}^{(8)}$ establish guidelines on maximum exposure levels for general public and workers, respectively. The former is based on action and limit values from the 1998 Guidelines for limiting exposure to time-varying EMF by International Commission on Non-Ionising Radiation Protection $(\text { ICNIRP })^{(9)}$. The latter is derived from action and limit values in the 2010 Guidelines for limiting exposure to time-varying EMF by ICNIRP ${ }^{(10)}$.

To ensure compliance with such frequency-dependent limits, recommendations and policies commonly describe measuring procedures to evaluate the field levels by means of EMF sensors ${ }^{(11,12)}$.

Broadband measurements with isotropic and true root-mean-square (RMS) field probes are often sufficient when they throw values far from reference levels. Conventional probes determine the RMS of the incident field strength on their partial sensors. Their root-sum-of-squares is compared with the minimum reference level at the frequency range of operation.

Narrowband measurements with fast-Fourier transform or superheterodyne spectrum analyzers are more convenient when broadband readings are close to reference levels. Omnidirectional antennas measure the field strength in three mutually perpendicular polarisations. Their root-sum-of-squares is compared with the reference level in the corresponding frequency band.

Regardless of the type of procedure carried out, broadband or narrowband, all measurement data are subject to some uncertainty and a measurement result must be given in association with some quantitative indication of its quality ${ }^{(13,14)}$. Without such an indication, measurement results cannot be compared, either among themselves or with reference levels.

The Guide to the Expression of Uncertainty in Measurement (GUM), originally published by the International Organization for Standardization (ISO) ${ }^{(15)}$, has been a staple in uncertainty analysis for nearly two decades $^{(16,17)}$. However, its inaccuracy when tackling non-linear systems and the inconsistency between Type A (frequentist) and Type B (Bayesian) assessment processes shown herein have lead to a vast variety of uncertainty analysis alternatives ${ }^{(18-21)}$. Supplement 1 to the GUM (GUMS1) was concerned to overcome its limitations by means of a Monte Carlo (MC) method suitable for non-linear models ${ }^{(22)}$. Alternatively, Bayesian-like approaches treat unknown parameters as random variables facilitating the combination of prior knowledge with information from observed data ${ }^{(23)}$.

Most authors have adopted the GUM framework for uncertainty analysis in EMF measurements ${ }^{(24-30)}$. 


\section{J. PRIETO ET AL.}

Only a few references concerning electromagnetic compatibility testing can be found following a GUMS1 approach ${ }^{(31,32)}$. Besides the aforementioned drawbacks, the assessment of EMF exposure implies two additional circumstances that require a different approach. First, the lack of inclusion of pertinent prior information may generate an uncertainty overestimation that requires the acquisition of additional measurements ${ }^{(12,33)}$. Secondly, the dynamic nature of propagation channel in urban, suburban, residential and rural areas may cause a misinterpretation of exposure risk when the involved distributions are modelled with static models ${ }^{(34-36)}$.

The aim of this study is 4-fold:

- to show the suitability of Bayesian perspective for the expression of uncertainty in the assessment of EMF exposure;

- to find prior information from measurement equipment or surrounding environment that may reduce such uncertainty;

- to dynamically adapt the involved distributions to observed data by non-parametric models such as kernel mixtures;

- to develop a generalised method for uncertainty analysis in EMF measurements that facilitates the integration of the above-mentioned points within a unified framework.

Notations: $f(x), F(x)$ and $\mathbb{E}\{x\}$ denote the probability density function (PDF), the probability distribution function and the expected value of the random variable $x$, respectively.

\section{MATERIALS AND METHODS}

The following sections describe conventional uncertainty propagation techniques in comparison with the proposed adaptive Bayesian framework for uncertainty analysis. Next, they likewise outline the experimental set-up along with the uncertainty components introduced by the EMF measurement equipment.

\section{Uncertainty propagation}

Conventional GUM and GUMS1 approaches are based on a model,

$$
y=g(\mathbf{x}),
$$

such that the information regarding the set of input quantities $\mathbf{x}=\left\{x_{n}\right\}_{n \in 1, \ldots, N}$ is propagated through the model to the output quantity of interest, $y$, in this case the EMF field strength (see Figure 1) ${ }^{(31)}$. The main difference between GUM and its Supplement lies in that the former propagates uncertainties through the model, whereas the latter propagates $\mathrm{PDFs}^{(37)}$.

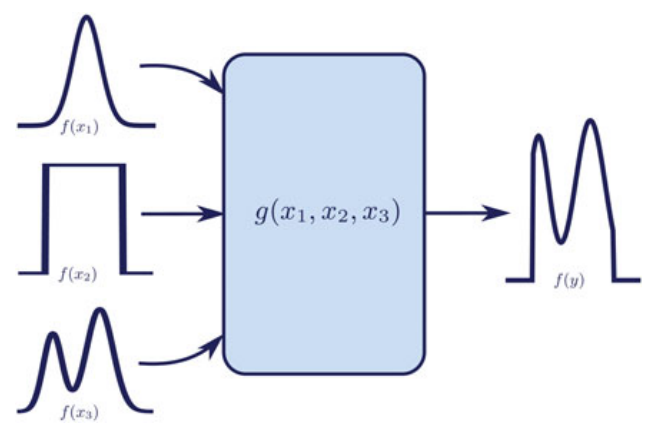

Figure 1. The Supplement 1 to the GUM obtains the PDFs for the input quantities and propagates them through the model to obtain the PDF for the output.

The basic steps for uncertainty propagation are summarised as follows ${ }^{(15,22)}$ :

- Formulation: develop a model, $y=g(\mathbf{x})$, relating the quantity intended to be measured, $y$, to the set of input quantities, $\mathbf{x}=\left\{x_{n}\right\}_{n \in 1, \ldots, N}$.

- Propagation: propagate the uncertainties, $u\left(x_{n}\right)$, or PDFs, $f\left(x_{n}\right)$, through the model to obtain the combined uncertainty, $u_{\mathrm{c}}(y)$, or $\operatorname{PDF}, f(y)$.

- Summarising: use the combined uncertainty, $u_{\mathrm{c}}(y)$, or PDF, $f(y)$, to obtain a coverage interval containing $y$ with a specified probability.

For the completion of the preceding steps, the GUM exploits the law of propagation of uncertainty, which expresses the idea that uncertainties in the inputs beget uncertainties in an output calculated from them. For example, in the case of $N$ uncorrelated input quantities, the law of propagation of uncertainty takes the form,

$$
u_{\mathrm{c}}^{2}(y)=\sum_{n=1}^{N}\left(\frac{\partial g}{\partial x_{n}}\right)^{2} u^{2}\left(x_{n}\right),
$$

where the partial derivatives are named sensitivity coefficients ${ }^{(38)}$. After obtaining the combined standard uncertainty, $u_{\mathrm{c}}(y)$, the GUM recommends to give an expanded uncertainty about the measurement result that encompasses a fraction $p$ of the density $f(y)$, characterised by such a result and its combined standard uncertainty. For practical considerations, the most common is to consider an interval $\left[y-k u_{\mathrm{c}}(y), y+k u_{\mathrm{c}}(y)\right]$. Note that if $f(y)$ is Gaussian and the effective number of degrees of freedom of $u_{\mathrm{c}}(y)$ is of significant size, $k=1.96$ and $k=2.58$ produce an interval with a confidence level of $\sim 95$ and $99 \%$, respectively.

For the same uncertainty computation, the GUMS1 utilises an alternative approach based on the 


\section{UNCERTAINTY ANALYSIS IN ELECTROMAGNETIC FIELD MEASUREMENTS}

$\mathrm{MC}$ method. It basically consists of randomly generating a number $M$ of MC trials in which a sample vector is drawn from the known PDFs of the inputs. Each drawn vector is propagated to the output through $g(\mathbf{x})$, obtaining a set of $M$ samples of $f(y)$. For example, in the case of $N$ independent input random variables, the $\mathrm{MC}$ method takes the form,

$$
\begin{gathered}
f(y)=\int_{-\infty}^{\infty} \ldots \int_{-\infty}^{\infty} f\left(x_{1}\right) \cdots f\left(x_{N}\right) \\
\delta\left(y-g\left(x_{1}, \ldots, x_{N}\right)\right) \mathrm{d} x_{1} \cdots \mathrm{d} x_{N} \\
\approx \frac{1}{M h} \sum_{m=1}^{M} K\left(\frac{y-g\left(x_{1}^{(m)}, \ldots, x_{N}^{(m)}\right)}{h}\right),
\end{gathered}
$$

where $\mathbf{x}_{m}=\left\{x_{n}^{(m)}\right\}_{n \in 1, \ldots, N}$ is a realisation of the set of input quantities $\mathbf{x}=\left\{x_{n}\right\}_{n \in 1, \ldots, N}{ }^{(39)}$. In Equation (3), $K(\cdot)$ is a function called kernel and $h$ is a positive number called bandwidth ${ }^{(40,41)}$. The GUMS1 utilises a uniform kernel to approximate $f(y)$ by a histogram; however, more flexible choices, such as a standard Gaussian kernel, can achieve better accuracies ${ }^{(42)}$. After obtaining the empirical PDF for $y, f(y)$, the GUMS1 seeks to provide a $100 p \%$ coverage interval from the distribution function, $F(y)$, with $p \in[0,1]$. From such a distribution, the coverage interval is given by $\left[F^{-1}(\alpha), F^{-1}(p+\alpha)\right]$, where $\alpha \in[0,1-p]$ and the quantile function,

$$
F^{(-1)}(\alpha)=\inf \{y \in \mathbb{R}: F(y) \geq \alpha\} .
$$

Uncertainty propagation methods are well suited to handle situations where there exists exact knowledge of the input quantities and their distributions, and of their relationship with the output quantity. However, such methods suffer from the absence of a unified framework to incorporate prior knowledge about the quantity of interest, in this case prior knowledge about the EMF distribution. Moreover, uncertainty propagation techniques lack a systematic methodology to dynamically adapt to observed data in a changing propagation environment. On the contrary, the Bayesian point of view is particularly advantageous to tackle such problems. As shown below, importance sampling under such an approach facilitates a generalised fusion of observed data and prior information. Moreover, the proposed methodology permits the use of more flexible choices to model observed data and prior information such as adaptive kernel mixtures.

\section{Bayesian uncertainty}

As stated in the GUM, one can assume that the combined standard uncertainty is a reliable estimate of possible error in the measurement if there is a sound basis that all influence factors are properly characterised $^{(15)}$. This section considers the Bayesian approach for optimal modelling of all error sources in the output, enabling the use of this modelling as a reliable measure of uncertainty.

Within the Bayesian framework, all model parameters involved in the measurement process are regarded as random variables, not as a description of their variability but as a description of the uncertainty about their true values ${ }^{(23)}$. Under such a paradigm, given a prior distribution, $f(y)$, and the likelihood function, $f(\mathbf{z} \mid y)$, the main task is to infer the posterior distribution, $f(y \mid \mathbf{z})$. The prior distribution expresses the uncertainty about the output, $y$, before seeing the data. The likelihood function conveys the information provided by the observed data, $\mathbf{z}$. The posterior distribution accounts for the uncertainty about the output, $y$, after seeing the data, $\mathbf{z}$.

The prior knowledge about the output can come from several avenues. Next sections describe different choices suitable for measuring the levels of exposure to EMF.

The likelihood function models the probability of the observed data given the field strength, $y$. Next sections develop an adaptive likelihood that considers both the measurement bias (systematic effects) and the dynamic nature (random errors) of the EMF measurements.

The posterior distribution summarises all the information regarding $y$ collected by the prior knowledge and the likelihood. From the Bayes' rule, the posterior density is immediately obtained as

$$
\begin{gathered}
f(y \mid \mathbf{z})=\frac{f(y) f(\mathbf{z} \mid y)}{\int f(y) f(\mathbf{z} \mid y) \mathrm{d} y}, \\
\alpha f(y) f(\mathbf{z} \mid y) .
\end{gathered}
$$

From $f(y \mid \mathbf{z})$, one can evaluate the measurement uncertainty where the most common is to provide the shortest $100 p \%$ credible interval or $100 p \%$ highest probability density interval, with $p \in[0,1]^{(22,43,44)}$. Calling $F(y \mid \mathbf{z})$ the posterior distribution function associated with $f(y \mid \mathbf{z})$, the shortest $100 p \%$ credible interval is $\left[F^{(-1)}(\alpha \mid \mathbf{z}), F^{(-1)}(p+\alpha \mid \mathbf{z})\right]$, where $\alpha$ is obtained from the quantile function as

$$
\begin{array}{cc}
\underset{\alpha \in[0,1]}{\arg \min } & \left\{F^{(-1)}(p+\alpha \mid \mathbf{z})-F^{(-1)}(\alpha \mid \mathbf{z})\right\} \\
\text { s.t. } & p \in[0,1], p+\alpha \in[0,1]
\end{array} .
$$

The basic steps of the Bayesian method for uncertainty analysis can be summarised as follows:

- Initialisation: obtain the prior PDF, $f(y)$. If the prior knowledge is conveyed by the input quantities, $\mathbf{x}=\left\{x_{n}\right\}_{n \in 1, \ldots, N}$, use the GUMS1 method to propagate the priors, $f\left(x_{n}\right)$, through the model to obtain $f(y)$. 


\section{J. PRIETO ET AL.}

- Update: correct the difference between $f(y)$ and $f(y \mid \mathbf{z})$ in light of the observed data via the likelihood, $f(\mathbf{z} \mid y)$.

- Summarising: use the posterior, $f(y \mid \mathbf{z})$, to obtain a coverage interval containing $y$ with a specified probability.

The resulting interval is often difficult to calculate analytically and it must be approximated numerically from samples of $f(y \mid \mathbf{z})$. However, it is possible to obtain samples from the posterior, $f(y \mid \mathbf{z})$, by implementing an importance sampling approach in which the prior, $f(y)$, plays the role of importance function $^{(45)}$. The idea behind this approach is to correct the difference between the posterior and the sampled prior by adding a weight to each sample. This weight is the result of evaluating the corresponding sample in the likelihood, $f(\mathbf{z} \mid y)$. Finally, a resampling step produces a set of $M$ samples, $\left\{y_{m}\right\}_{m \in 1, \ldots, M}$, that leads to an approximation to the posterior of the form,

$$
f(y \mid \mathbf{z}) \approx \frac{1}{M h} \sum_{m=1}^{M} K\left(\frac{y-y_{m}}{h}\right),
$$

from which it is straightforward to obtain the $100 \mathrm{p} \%$ credible interval in a similar fashion to GUMS1. A possible implementation of resampling can be the generation of a sample from the uniform distribution $\mathcal{U}(0,1)$ and the selection of $y_{\mathrm{c}}$ such that the generated sample is greater than the cumulative sum of the weights $\left\{\omega_{m}\right\}_{m \in 1, \ldots, M}$ up to $c$ but not up to $(c-1)^{(45)}$.

If sampling from the prior distribution is not possible, the use of an importance distribution, $\pi(y)$, allows drawing samples and obtaining weights as the algorithm in Appendix A shows. Note that the proposed implementation avoids the computation of the normalising constant in Equation (5). In order to implement such an algorithm, it is mandatory to completely define the prior density and the likelihood function. Next two sections develop such models.

\section{Prior information for EMF measurements}

As aforementioned, the additional information provided by prior knowledge contributes to better interpret the risks of exposure to EMF and avoid unnecessary extra measurements. Such prior knowledge can come from several avenues, e.g. vague knowledge, manufacturer specifications or past experiences. This study considers the following types of prior distributions:

(1) Reference prior: a default no informative prior that is the same as the Jeffreys' prior for the presented univariate problem $^{(33)}$. The Jeffreys' prior is defined as,

$$
f(y) \alpha \sqrt{\mathcal{I}(y)},
$$

where $\mathcal{I}(y)$ is the Fisher information given by the variance of the score function:

$$
\mathcal{I}(y)=-\mathbb{E}\left\{\frac{\partial^{2} \log f(\mathbf{z} \mid y)}{\partial y^{2}}\right\} .
$$

(1) Vague prior: a prior density of high spread that incorporates vague knowledge such as the range of operation of the measuring device. For example, if the manufacturer specifications provide lower and upper limits of operation, $a$ and $b$, respectively, the vague prior can be given by a uniform distribution:

$$
f(y)=\left\{\begin{array}{cc}
\frac{1}{b-a} & \text { for } a \leq y \leq b \\
0 & \text { otherwise }
\end{array}\right.
$$

(2) Informative prior: a prior that conveys information from the modelling of the propagation channel and EMF sources or from historical EMF data collected in a similar environment to the one being assessed. For example, an informative prior may harbor statements such as 'the received EMF strength at a ground level in urban areas generally falls below a given threshold' or 'the measured EMF strength in the vicinity of schools is commonly under a certain level'. The former requires an exhaustive study of all influence factors in a three-dimensional space that is neither cost-effective nor resilient to environmental changes ${ }^{(46)}$. The latter accommodates prior distributions from measurements performed in analogous areas (urban, rural, etc.), inside the same facility type (hospital, school, etc.), and with similar surroundings (transmitting antennas, skyscrapers, etc. $)^{(35)}$. Following this second approach, this study fits historical data stored in the same facility with a three-component Gaussian mixture. A three-component Gaussian mixture is well suited for EMF strength measurements since each component corresponds to a different type of traffic (at night, working or rush hours) ${ }^{(47)}$. The data consisted of thousands of uncorrelated values measured in tens of points during different time slots. Such data were fitted with the ExpectationMaximization (EM) algorithm with starting values obtained through $k$-means clustering ${ }^{(48)}$,

$$
f(y)=\sum_{i=1}^{3} \frac{\omega_{i}}{\sqrt{2 \pi} \sigma_{i}} \exp \left(-\frac{\left(y-\mu_{i}\right)^{2}}{2 \sigma_{i}^{2}}\right) .
$$


Adaptive likelihood for EMF measurements

Conventional uncertainty budgets in EMF measurements include an uncertainty component accounting for the random error (or repeatability) in the measurements. This component has traditionally been modelled by a zero-mean Gaussian distribution $(28,32)$ or neglected $^{(25)}$. However, these assumptions do not hold for small sample sizes or fast-changing propagation environments. Lognormal, Rice, Rayleigh, Suzuki, Nakagami, Weibull, extreme-value or Gaussian mixture distributions have demonstrated to fit more accurately fading data in urban, suburban or indoor areas ${ }^{(47,49-51)}$. As illustrated in the following, the random error contribution to uncertainty is better captured by a non-parametric approach based on a mixture of kernels.

Let $z$ be the measured EMF strength in a given time instant and the measurements, $\mathbf{z}=\left\{z_{i}\right\}_{i \in 1, \ldots, S}$, a set of $S$ i.i.d. samples from such a random variable and assume that the set of measurements is obtained under the same propagation conditions, method and equipment. Consequently, the $S$ measurements are affected by the same EMF strength, $y$, and systematic effects, $b$, that may change for any set of samples collected in a different time instant if measurement conditions, method or equipment is altered ${ }^{(12)}$. Calling $\overline{\mathbf{z}}$ the sample mean of the set of measurements, the likelihood function of the EMF strength can be approximated as (see Appendix B)

$$
\begin{aligned}
& f(\mathbf{z} \mid y), \\
\propto & \mathbb{E}_{b}\left\{\prod_{j=1}^{S} \sum_{i=1}^{S} \exp \left(-\frac{\left(z_{j}-z_{i}+\overline{\mathbf{z}}-y-b\right)^{2}}{2 h^{2}}\right)\right\} .
\end{aligned}
$$



Figure 2. Measurements taken both outside and inside an office building recreated common circumstances found during a typical campaign of assessment of exposure levels to EMF. 
J. PRIETO ET AL.

Table 1. Uncertainties associated with broadband measurements.

\begin{tabular}{lcc}
\hline Influence factor & PDF [dB] & $U\left(x_{n}\right)[\mathrm{dB}]$ \\
\hline $\begin{array}{l}\text { Whole system } \\
\text { Repeatability of indication }{ }^{\mathrm{a}}\end{array}$ & $\mathcal{N}(0.00, s(\mathbf{z}) / \sqrt{S})$ & $s(\mathbf{z}) / \sqrt{S}$ \\
Radiation meter & $\mathcal{U}(-0.07,0.07)$ & 0.04 \\
$\quad$ Resolution & $\mathcal{U}(-1.00,1.00)$ & 0.58 \\
$\quad$ Modulation error & $\mathcal{U}(-2.05,2.73)$ & 1.38 \\
Probe & $\mathcal{U}(-0.83,0.83)$ & 0.48 \\
$\quad$ Frequency response & $\mathcal{U}(-0.18,0.26)$ & 0.13 \\
$\quad$ Magnetic field suppression & $\mathcal{U}(-0.05,0.05)$ & 0.03 \\
$\quad$ Non-linearity & $\mathcal{U}(-1.50,0.20)$ & 0.49 \\
Anisotropy & & \\
Temperature variation & $\mathcal{U}(-0.83,0.83)$ & 0.48 \\
Location & & \\
Coupling from nearby objects & & \\
\hline
\end{tabular}

The term $s(\cdot)$ accounts for the sample standard deviation, while $S$ denotes the number of measurements.

${ }^{a}$ In the proposed Bayesian uncertainty analysis, the repeatability of indication is modelled with the adaptive density, $f(\mathbf{z} \mid y, b)$, in Appendix B.

Fresnel zone clearance. As Figure 2 reflects, two mobile phone base stations were emitting 260 and $430 \mathrm{~m}$ away from the building, respectively. Outdoor points and one of the indoor points were in line-ofsight to base stations. All indoor points were collected on the second floor $(15 \mathrm{~m}$ from the ground) where there were three IEEE 802.11 access points along the corridor. In the following, two cases are considered:

- Individual measurements: measurements collected at Points 1-6 in Figure 2 and sampled with the minimum time interval, $0.4 \mathrm{~s}$, to guarantee similar conditions $^{(52)}$.

- Averaged measurements: measurements stored at Point 7 in Figure 2 and averaged over the recommended 0.1 -h interval $^{(9)}$. Each averaged value is obtained by the radiation meter from a different set of measurements, guaranteeing the independence among averaged measurements.

\section{Uncertainty contributions in EMF measurements}

The most relevant contributions to the uncertainty budget in broadband measurements are associated with the repeatability of indication, the radiation meter, the probe and the location. Except for the repeatability of indication, which accounts for the random error, all other influence factors will be treated as systematic effects $^{(12)}$. The results of this study are obtained by assuming independence among all the uncertainty components contributing to the bias. This represents a mild assumption since the thorough selection of measurement equipment and procedure can ensure that possible dependencies are avoided or minimised. Table 1 summarises such contributions that are presented afterwards.
Uncertainties associated with the repeatability of indication gather all random errors in a set of measurements collected in a short distance of time, under the same propagation conditions, by the same operator and with the same instrumentation. The repeatability is then connected with the sole source of random uncertainty. This type of uncertainty is encoded by the density $f(\mathbf{z} \mid y, b)$, typically a zero-mean Gaussian (a kernel mixture in this study).

Uncertainties associated with the radiation meter are as follows:

- Resolution: the uncertainty is a function of the least significant digit in the display readout or the ability to distinguish between two close but different values of the measurand ${ }^{(15)}$. It is usually modelled by means of a uniform distribution of length the resolution of the radiation meter centred at zero.

- Modulation error: the waveform of the field source may provoke the diode detector to leave the square-law region when driven at higher signal levels and no longer operate as a true RMS rectifier. The distribution of this component is commonly assumed to be uniform with boundaries obtained from manufacturer tests ${ }^{(53)}$.

Uncertainties associated with the probe are as follows:

- Frequency response: the flatness within the frequency range of operation becomes a key factor in situations with emissions from multiple or unknown frequencies (in such cases, it is not possible to correct the measurement through correction factors). In this study, a uniform distribution models the worst flatness in frequency as a contributor to the measurement uncertainty. 
Table 2. Uncertainty analysis for $S$ individual measurements from GUM, GUMS1 and Bayesian perspectives.

\begin{tabular}{|c|c|c|c|c|c|c|c|c|c|c|c|c|c|}
\hline \multirow[t]{2}{*}{ Point } & \multirow[t]{2}{*}{ Method } & \multicolumn{3}{|c|}{$S=5$} & \multicolumn{3}{|c|}{$S=10$} & \multicolumn{3}{|c|}{$S=50$} & \multicolumn{3}{|c|}{$S=100$} \\
\hline & & $\hat{y}$ & $u_{c}(y)$ & $95 \%$ c.i. & $\hat{y}$ & $u_{c}(y)$ & $95 \%$ c.i. & $\hat{y}$ & $u_{c}(y)$ & $95 \%$ c.i. & $\hat{y}$ & $u_{c}(y)$ & $95 \%$ c.i. \\
\hline 1 & $\begin{array}{l}\text { GUM } \\
\text { GUMS1 } \\
\text { Reference } \\
\text { Vague } \\
\text { Informative }\end{array}$ & $\begin{array}{l}4.59 \\
4.60 \\
4.49 \\
4.40 \\
4.37\end{array}$ & $\begin{array}{l}1.72 \\
1.73 \\
1.68 \\
1.65 \\
1.10\end{array}$ & $\begin{array}{l}1.22,7.96 \\
1.36,7.82 \\
1.26,7.61 \\
1.09,7.39 \\
2.19,6,41\end{array}$ & $\begin{array}{l}4.51 \\
4.54 \\
4.56 \\
4.59 \\
4.65\end{array}$ & $\begin{array}{l}1.72 \\
1.73 \\
1.69 \\
1.66 \\
1.29\end{array}$ & $\begin{array}{l}1.14,7.89 \\
1.32,7.78 \\
1.12,7.55 \\
1.40,7.71 \\
1.89,6.09\end{array}$ & $\begin{array}{l}4.29 \\
4.28 \\
4.27 \\
4.27 \\
4.65\end{array}$ & $\begin{array}{l}1.72 \\
1.73 \\
1.66 \\
1.67 \\
1.24\end{array}$ & $\begin{array}{l}0.92,7.66 \\
1.01,7.51 \\
1.22,7.35 \\
1.22,7.39 \\
1.95,5.97\end{array}$ & $\begin{array}{l}4.29 \\
4.29 \\
4.35 \\
4.30 \\
4.23\end{array}$ & $\begin{array}{l}1.72 \\
1.73 \\
1.70 \\
1.68 \\
1.20\end{array}$ & $\begin{array}{l}0.92,7.66 \\
1.04,7.59 \\
1.31,7.54 \\
1.32,7.53 \\
2.05,6.54\end{array}$ \\
\hline 2 & $\begin{array}{l}\text { GUM } \\
\text { GUMS1 } \\
\text { Reference } \\
\text { Vague } \\
\text { Informative }\end{array}$ & $\begin{array}{l}4.94 \\
4.93 \\
4.88 \\
4.83 \\
4.73\end{array}$ & $\begin{array}{l}1.72 \\
1.72 \\
1.64 \\
1.60 \\
1.18\end{array}$ & $\begin{array}{l}1.56,8.32 \\
1.68,8.16 \\
1.87,7.93 \\
1.87,7.89 \\
2.06,6.01\end{array}$ & $\begin{array}{l}5.02 \\
5.02 \\
5.06 \\
5.00 \\
4.78\end{array}$ & $\begin{array}{l}1.72 \\
1.72 \\
1.65 \\
1.65 \\
1.16\end{array}$ & $\begin{array}{l}1.65,8.39 \\
1.83,8.26 \\
2.17,8.35 \\
2.04,8.09 \\
2.11,6.14\end{array}$ & $\begin{array}{l}5.15 \\
5.14 \\
5.04 \\
5.09 \\
4.59\end{array}$ & $\begin{array}{l}1.72 \\
1.73 \\
1.65 \\
1.62 \\
1.09\end{array}$ & $\begin{array}{l}1.77,8.52 \\
1.80,8.31 \\
1.98,8.19 \\
2.42,8.03 \\
2.59,6.72\end{array}$ & $\begin{array}{l}5.16 \\
5.16 \\
5.12 \\
5.10 \\
4.64\end{array}$ & $\begin{array}{l}1.72 \\
1.73 \\
1.71 \\
1.71 \\
1.14\end{array}$ & $\begin{array}{l}1.79,8.53 \\
1.92,8.43 \\
2.08,8.38 \\
2.01,8.15 \\
2.55,6.86\end{array}$ \\
\hline 3 & $\begin{array}{l}\text { GUM } \\
\text { Reference } \\
\text { Vague } \\
\text { Informative }\end{array}$ & $\begin{array}{l}2.21 \\
2.12 \\
2.12 \\
3.48\end{array}$ & $\begin{array}{l}1.74 \\
1.65 \\
1.64 \\
1.12\end{array}$ & $\begin{array}{r}1.19,5.61 \\
-0.96,5.22 \\
-0.76,5.25 \\
1.39,5.51\end{array}$ & $\begin{array}{l}2.00 \\
1.92 \\
1.89 \\
3.31\end{array}$ & $\begin{array}{l}1.72 \\
1.68 \\
1.71 \\
1.14\end{array}$ & $\begin{array}{r}-1.38,5.38 \\
-0.98,5.24 \\
-1.11,5.26 \\
1.17,5.53\end{array}$ & $\begin{array}{l}1.97 \\
1.89 \\
1.77 \\
3.29\end{array}$ & $\begin{array}{l}1.72 \\
1.67 \\
1.65 \\
1.14\end{array}$ & $\begin{array}{r}-1.40,5.34 \\
-1.28,4.99 \\
-1.38,4.99 \\
1.30,5.37\end{array}$ & $\begin{array}{l}1.91 \\
1.83 \\
1.75 \\
3.20\end{array}$ & $\begin{array}{l}1.72 \\
1.70 \\
1.70 \\
1.23\end{array}$ & $\begin{array}{r}-1.46,5.28 \\
-1.18,5.20 \\
-1.22,5.28 \\
1.07,5.53\end{array}$ \\
\hline 4 & $\begin{array}{l}\text { GUM } \\
\text { GUMS1 } \\
\text { Reference } \\
\text { Vague } \\
\text { Informative }\end{array}$ & $\begin{array}{l}-8.22 \\
-8.21 \\
-8.20 \\
-8.22 \\
-8.46\end{array}$ & $\begin{array}{l}1.86 \\
1.85 \\
1.73 \\
1.70 \\
1.58\end{array}$ & $\begin{array}{l}-11.86,-4.57 \\
-11.73,-4.64 \\
-11.45,-5.04 \\
-10.92,-4.74] \\
-11.31,-5.33\end{array}$ & $\begin{array}{l}-8.11 \\
-8.12 \\
-8.09 \\
-8.07 \\
-8.35\end{array}$ & $\begin{array}{l}1.77 \\
1.76 \\
1.73 \\
1.74 \\
1.59\end{array}$ & $\begin{array}{l}-11.59,-4.64 \\
-11.44,-4.76 \\
-11.41,-5.02 \\
-11.17,-4.80 \\
-15.16,-9.26\end{array}$ & $\begin{array}{l}-6.88 \\
-6.85 \\
-6.92 \\
-6.92 \\
-7.27\end{array}$ & $\begin{array}{l}1.73 \\
1.73 \\
1.67 \\
1.62 \\
1.59\end{array}$ & $\begin{array}{r}-10.26,-3.50 \\
-10.14,-3.61 \\
-10.17,-3.73 \\
-10.16,-4.20 \\
-9.76,-4.09\end{array}$ & $\begin{array}{l}-6.33 \\
-6.33 \\
-6.29 \\
-6.28 \\
-6.68\end{array}$ & $\begin{array}{l}1.72 \\
1.73 \\
1.75 \\
1.73 \\
1.65\end{array}$ & $\begin{array}{l}-9.71,-2.96 \\
-9.59,-3.10 \\
-9.45,-3.17 \\
-9.62,-3.29 \\
-9.79,-3.78\end{array}$ \\
\hline 5 & $\begin{array}{l}\text { GUM } \\
\text { GUMS1 } \\
\text { Reference } \\
\text { Vague } \\
\text { Informative }\end{array}$ & $\begin{array}{l}-11.08 \\
-11.08 \\
-11.21 \\
-11.22 \\
-10.55\end{array}$ & $\begin{array}{l}1.92 \\
1.92 \\
1.70 \\
1.68 \\
1.41\end{array}$ & $\begin{array}{l}-14.85,-7.31 \\
-14.77,-7.38 \\
-14.18,-8.09 \\
-14.09,-8.09 \\
-13.30,-7.93\end{array}$ & $\begin{array}{l}-13.02 \\
-13.03 \\
-13.01 \\
-13.02 \\
-12.11\end{array}$ & $\begin{array}{l}2.01 \\
2.02 \\
1.77 \\
1.75 \\
1.57\end{array}$ & $\begin{array}{l}-16.95,-9.09 \\
-16.93,-9.15 \\
-16.07,-9.88 \\
-15.98,-9.96 \\
-15.16,-9.26\end{array}$ & $\begin{array}{l}-14.28 \\
-14.67 \\
-14.58 \\
-14.52 \\
-13.71\end{array}$ & $\begin{array}{l}1.78 \\
1.77 \\
1.69 \\
1.68 \\
1.66\end{array}$ & $\begin{array}{l}-17.76,-10.80 \\
-18.06,-11.36 \\
-17.70,-11.71 \\
-17.59,-11.70 \\
-16.48,-10.54\end{array}$ & $\begin{array}{l}-14.24 \\
-14.24 \\
-14.26 \\
-14.33 \\
-13.51\end{array}$ & $\begin{array}{l}1.74 \\
1.74 \\
1.69 \\
1.67 \\
1.44\end{array}$ & $\begin{array}{l}-17.66,-10.83 \\
-17.53,-10.98 \\
-17.52,-11.16 \\
-17.83,-11.55 \\
-16.35,-10.95\end{array}$ \\
\hline 6 & $\begin{array}{l}\text { GUM } \\
\text { GUMS1 } \\
\text { Reference } \\
\text { Vague } \\
\text { Informative }\end{array}$ & $\begin{array}{l}-9.81 \\
-9.80 \\
-9.93 \\
-9.88 \\
-9.89\end{array}$ & $\begin{array}{l}2.17 \\
2.15 \\
1.68 \\
1.67 \\
1.58\end{array}$ & $\begin{array}{l}-14.07,-5.55 \\
-14.05,-5.71 \\
-13.11,-6.97 \\
-12.94,-6.97 \\
-12.61,-6.80\end{array}$ & $\begin{array}{l}-13.25 \\
-13.25 \\
-13.21 \\
-13.29 \\
-12.09\end{array}$ & $\begin{array}{l}3.74 \\
3.74 \\
1.60 \\
1.56 \\
1.45\end{array}$ & $\begin{array}{l}-20.58,-5.92 \\
-20.53,-5.95 \\
-16.73,-10.79 \\
-16.32,-10.80 \\
-14.88,-9.59\end{array}$ & $\begin{array}{l}-15.84 \\
-15.87 \\
-15.71 \\
-15.68 \\
-14.84\end{array}$ & $\begin{array}{l}2.00 \\
2.00 \\
1.70 \\
1.70 \\
1.58\end{array}$ & $\begin{array}{l}-19.76,-11.91 \\
-19.72,-12.06 \\
-18.89,-12.75 \\
-18.76,-12.31 \\
-18.11,-12.21\end{array}$ & $\begin{array}{l}-16.13 \\
-16.15 \\
-16.14 \\
-16.16 \\
-15.11\end{array}$ & $\begin{array}{l}1.87 \\
1.88 \\
1.66 \\
1.63 \\
1.51\end{array}$ & $\begin{array}{l}-19.79,-12.47 \\
-19.66,-12.46 \\
-19.37,-13.13 \\
-19.32,-13.08 \\
-18.58,-12.81\end{array}$ \\
\hline
\end{tabular}

c.i. denotes confidence interval or credible interval, as applicable. Estimated means and uncertainties are in $\mathrm{dBV} \mathrm{m}^{-1}$ and $\mathrm{dB}$, respectively. 


\section{J. PRIETO ET AL.}

- Magnetic field suppression: in the E-field mode, the contribution of the undesired magnetic field components must be eliminated. Without additional information, this uncertainty component is modelled as a uniform random variable of length reported in the specification ${ }^{(52)}$.

- Non-linearity: the variability in the response of the radiation meter to changes in the E-field strength is closely tied to the diode detector. Such variability is assumed to follow a uniform distribution limited by the minimum and maximum linearity correction factors provided in the calibration certificate.

- Anisotropy: the anisotropy is the maximum deviation from the radiation pattern of an isotropic antenna $^{(54)}$. It is commonly represented by a uniform distribution parametrised by values specified in the calibration certificate.

- Temperature variation: the technical specification of the sensor reports the highest deviation related to the minimum and maximum temperature of operation $^{(52)}$. This component is characterised by a uniform distribution limited by such a deviation.

Uncertainties associated with the location of the probe arise when conducting objects or high-dielectricconstant elements are placed near the point of measurement ${ }^{(11)}$. Objects such as metal fences, motor vehicles or house wiring play the role of reradiators or scatterers that alter the impedance match between the probe's antenna and its detector. Under the described measuring conditions, if the distance between the field probe and small perturbing objects is $>20 \mathrm{~cm}$ the contribution of this uncertainty component is no $>10 \%$ of the measured EMF strength ${ }^{(11)}$. Such value provides the semi-amplitude of the uniform distribution that conveys this uncertainty contribution.

\section{RESULTS AND DISCUSSION}

This section compares the performance of the uncertainty analyses provided by GUM, GUMS1 and the proposed adaptive Bayesian approach. It first assesses the case of reporting the uncertainty from a set of individual measurements. Afterwards, it provides a parallel study when the measurements are averaged over the recommended 0.1-h interval.

\section{Individual measurements}

In order to illustrate how the proposed framework operates, Table 2 summarises the results of an uncertainty analysis carried out at Points 1-6 in Figure 2. It includes the expected value, standard deviation and $95 \%$ coverage intervals estimated with $5,10,50$ and 100 measurements. It calls:

- GUM: the conventional uncertainty analysis detailed at GUM. All sensitivity coefficients were set to 1 and the coverage factor to $k=1.96^{(15)}$. Table 2 reports confidence intervals for the most optimistic case in which the coverage factor is obtained from a Gaussian distribution. The Student's $t$-distribution would provide wider intervals for small sample sizes.

- GUMS1: the MC approach outlined in the Supplement 1 to the GUM. The number of MC experiments was fixed to $10000^{(22)}$.

- Reference: the proposed adaptive framework based on the Jeffreys' prior. For the sake of simplicity, the Jeffreys' prior was obtained considering a Gaussian random error and a uniform systematic error. The importance sampling was accomplished with 10000 MC realisations (see Appendix A).

- Vague: the proposed adaptive framework with a uniform prior that models the range of operation of the measurement equipment. The importance sampling was carried out with $2000 \mathrm{MC}$ trials (see Appendix A).

- Informative: the proposed adaptive framework with a prior mixture obtained by the EM algorithm above explained. The importance sampling was performed with $1000 \mathrm{MC}$ experiments (see Appendix A).

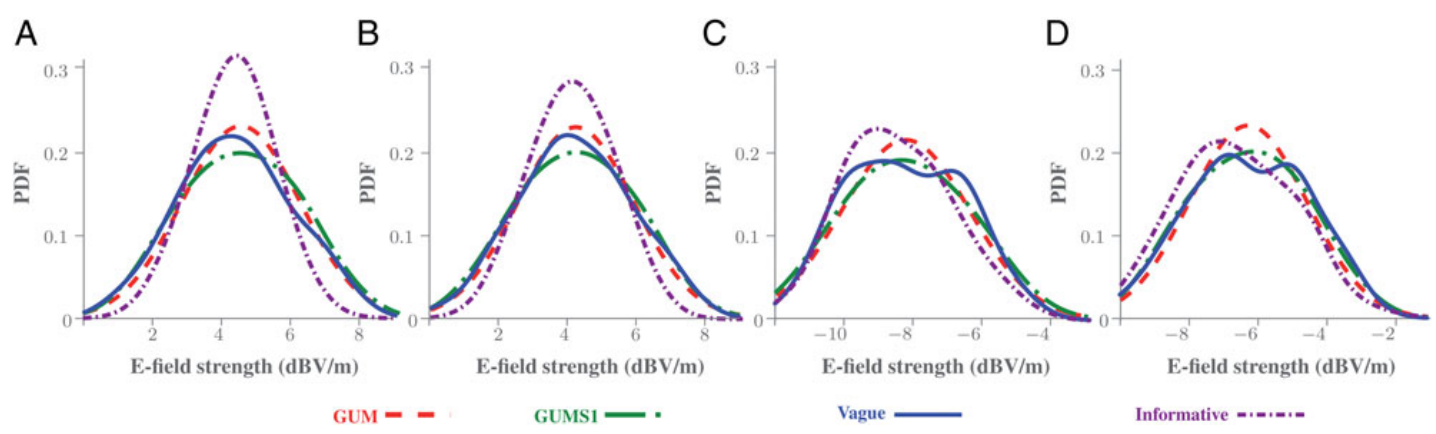

Figure 3. The dynamic nature of the EMF is better captured by the proposed adaptive uncertainty analysis performed with: (A) 5 individual measurements in an outdoor environment (Point 1); (B) 100 individual measurements in an outdoor environment (Point 1); (C) 5 individual measurements in an indoor environment (Point 4); (D) 100 individual measurements in an indoor environment (Point 4). 
Table 3. Uncertainty analysis for $S$ averaged measurements from GUM, GUMS1 and Bayesian perspectives.

\begin{tabular}{|c|c|c|c|c|c|c|c|c|c|c|c|c|c|}
\hline \multirow[t]{2}{*}{ Time } & \multirow[t]{2}{*}{ Method } & \multicolumn{3}{|c|}{$S=5$} & \multicolumn{3}{|c|}{$S=10$} & \multicolumn{3}{|c|}{$S=50$} & \multicolumn{3}{|c|}{$S=100$} \\
\hline & & $\hat{y}$ & $u_{\mathrm{c}}(y)$ & $95 \%$ c.i. & $\hat{y}$ & $u_{\mathrm{c}}(y)$ & $95 \%$ c.i. & $\hat{y}$ & $u_{\mathrm{c}}(y)$ & $95 \%$ c.i. & $\hat{y}$ & $u_{\mathrm{c}}(y)$ & $95 \%$ c.i. \\
\hline \multirow[t]{5}{*}{8 a.m. -10 a.m. } & GUM & -5.26 & 1.82 & $-8.82,-1.69$ & -4.89 & 1.75 & $-8.32,-1.46$ & -3.97 & 1.72 & $-7.35,-0.59$ & -3.61 & 1.72 & $-6.98,-0.23$ \\
\hline & GUMS1 & -5.25 & 1.82 & $-8.71,-1.76$ & -4.88 & 1.73 & $-8.20,-1.63$ & -3.97 & 1.71 & $-7.21,-0.77$ & -3.62 & 1.71 & $-6.83,-0.36$ \\
\hline & Reference & -5.31 & 1.74 & $-8.51,-2.31$ & -4.77 & 1.69 & $-7.87,-1.48$ & -4.00 & 1.66 & $-7.31,-0.98$ & -3.66 & 1.71 & $-6.54,-0.15$ \\
\hline & Vague & -5.37 & 1.70 & $-8.73,-2.31$ & -4.82 & 1.63 & $-7.87,-1.59$ & -4.04 & 1.66 & $-7.30,-0.95$ & -3.65 & 1.69 & $-6.57,-0.15$ \\
\hline & Informative & -6.04 & 1.52 & $-8.50,-2.84$ & -5.59 & 1.63 & $-8.77,-2.71$ & -4.89 & 1.46 & $-7.43,-2.12$ & -4.60 & 1.49 & $-7.31,-1.97$ \\
\hline \multirow[t]{5}{*}{12 p.m. -2 p.m. } & GUM & -2.84 & 1.72 & $-6.22,0.54$ & -2.84 & 1.72 & $-6.21,0.53$ & -3.57 & 1.72 & $-6.94,-0.19$ & -3.71 & 1.72 & $-7.08,-0.34$ \\
\hline & GUMS1 & -2.86 & 1.73 & $-6.08,0.39$ & -2.84 & 1.73 & $-6.09,0.39$ & -3.58 & 1.73 & $-6.80,-0.27$ & -3.73 & 1.71 & $-6.95,-0.51$ \\
\hline & Reference & -2.97 & 1.73 & $-5.99,0.20$ & -2.90 & 1.69 & $-6.00,0.18$ & -3.55 & 1.69 & $-6.71,-0.56$ & -3.70 & 1.69 & $-7.04,-0.51$ \\
\hline & Vague & -3.03 & 1.69 & $-6.05,0.00$ & -2.93 & 1.70 & $-5.97,0.15$ & -3.54 & 1.65 & $-6.71,-0.56$ & -3.75 & 1.70 & $-6.85,-0.48$ \\
\hline & Informative & -2.93 & 1.68 & $-6.16,-0.22$ & -3.83 & 1.58 & $-6.63,-0.99$ & -4.44 & 1.57 & $-7.12,-1.44$ & -4.64 & 1.42 & $-7.19,-1.74$ \\
\hline \multirow[t]{5}{*}{ Mixed } & GUM & -3.65 & 1.73 & $-7.04,-0.26$ & -3.32 & 1.73 & $-6.70,0.06$ & -3.74 & 1.72 & $-7.12,-0.36$ & -3.58 & 1.72 & $-6.95,-0.21$ \\
\hline & GUMS1 & -3.67 & 1.74 & $-6.94,-0.40$ & -3.31 & 1.72 & $-6.61,-0.10$ & -3.74 & 1.73 & $-6.98,-0.45$ & -3.59 & 1.73 & $-6.80,-0.33$ \\
\hline & Reference & -3.80 & 1.69 & $-6.92,-0.58$ & -3.31 & 1.69 & $-6.24,0.03$ & -3.64 & 1.70 & $-6.98,-0.45$ & -3.52 & 1.70 & $-6.96,-0.59$ \\
\hline & Vague & -3.78 & 1.67 & $-6.84,-0.62$ & -3.33 & 1.69 & $-6.63,-0.43$ & -3.71 & 1.68 & $-6.93,-0.43$ & -3.55 & 1.68 & $-6.90,-0.64$ \\
\hline & Informative & -4.07 & 1.56 & $-7.38,-1.36$ & -3.51 & 1.66 & $-6.88,-0.82$ & -3.87 & 1.65 & $-7.14,-0.97$ & -3.66 & 1.67 & $-7.12,-1.11$ \\
\hline
\end{tabular}

c.i. denotes confidence interval or credible interval, as applicable. Estimated means and uncertainties are in $\mathrm{dBV} \mathrm{m}^{-1}$ and $\mathrm{dB}$, respectively. 


\section{J. PRIETO ET AL.}

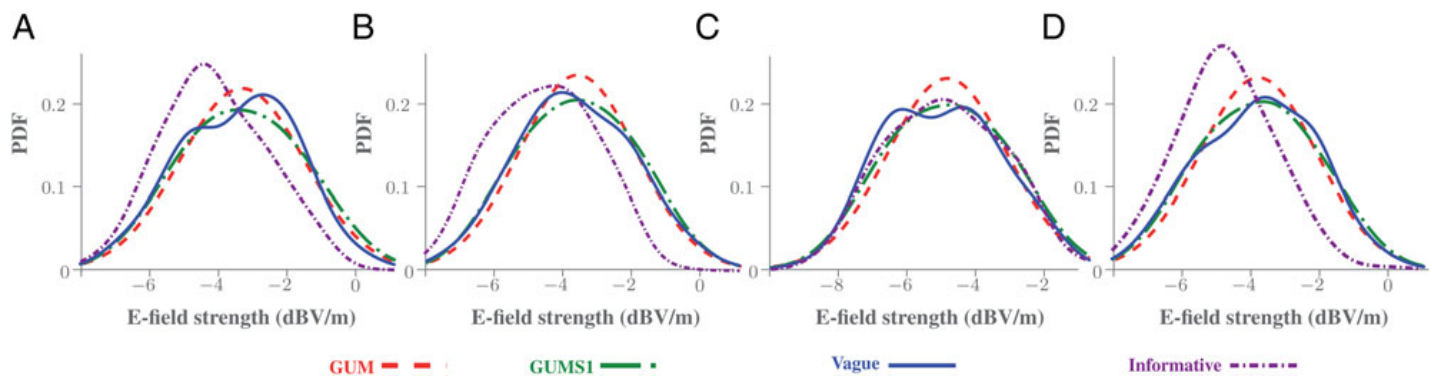

Figure 4. The dynamic nature of the EMF is also better explained by the proposed adaptive uncertainty analysis performed in the indoor Point 7 with: (A) 5 averaged measurements during working hours; (B) 100 averaged measurements during working hours; (C) 5 averaged measurements during rush hours; (D) 100 averaged measurements during rush hours.

The three Bayesian analyses approximated the likelihood with 5000 MC samples (see Appendix C). Notice that in natural units the greatest difference among all mean values in Table 2 is $0.23 \mathrm{~V} \mathrm{~m}^{-1}$ and that differences between reference and vague priors come from approximation. For large sample sizes, such high-spread priors offer similar results to GUMS1 due to the reduction of the uncertainty associated with repeatability.

Figure 3 illustrates the density functions utilised by the above methods to calculate the uncertainty, i.e. a Gaussian density for the GUM approach and a Gaussian mixture for the MC sampling methods. Note that Figure 3 does not include the posterior density function obtained with the reference prior due to its similarity to the result achieved with the vague prior.

From Table 2 and Figure 3 one can point out that: (1) the proposed adaptive likelihood handles the dynamic nature of EMF measurements in a better fashion than conventional GUM and GUMS1 methods, particularly in indoor areas; (2) the fusion of such likelihood with prior information always produces mean estimates with a remarkable reduction of measurement uncertainty, especially for small sample sizes and (3) these improvements result in an overall reduction in GUM and GUMS1 $95 \%$ coverage intervals $>11 \%$ when using vague or reference priors, and $>28 \%$ when applying informative priors. Moreover, vague priors achieve a performance similar to that with reference priors and reduce the number of samples required to approximate the posterior.

\section{Averaged measurements}

Analogously to the uncertainty analysis for individual measurements, Table 3 contains the results for averaged measurements collected at Point 7 in Figure 2. It gathers the same set of uncertainty analyses performed in previous section. In order to reproduce realistic conditions and assure that all averaged data are collected under similar conditions, the following three types of measurements are considered:
- 8 a.m.-10 a.m.: measurements collected from 8 a.m. to 10 a.m. in five different working-days to emulate the traffic in working hours.

- 12 p.m.-2 p.m.: measurements taken from 12 p.m. (noon) to 2 p.m. in five different working-days to recreate the traffic in rush hours.

- Mixed: data sampled with replacement from a set of 100 measurements from each type of traffic to simulate areas with changing EMF sources.

Notice that in natural units the greatest difference among all mean values in Table 3 is $0.08 \mathrm{~V} \mathrm{~m}^{-1}$.

In concordance with previous section, Figure 4 displays the density functions utilised by the assessed uncertainty analysis methods.

In light of Table 3 and Figure 4, one can conclude that: (1) averaging the measurements reduces their variability, however, the proposed adaptive likelihood still reflects their residual dynamic behavior, significantly appreciable for working traffic; (2) the reduction in measurement uncertainty is more noticeable if such likelihood is combined with an informative prior, independently of the sample size and (3) the use of the proposed likelihood dwarfs the limits of GUM and GUMS1 $95 \%$ coverage intervals >4\% when endowed with vague and reference priors, and $>11 \%$ when coupled with informative priors. Furthermore, estimating posterior distributions with the proposed approach facilitates a more natural interpretation of uncertainty credible intervals since the latter contain the variable of interest with the specified probability.

\section{CONCLUSION}

This study has presented a new framework to deal with uncertainty in EMF measurements. Such a framework dynamically adapts to measurement data, which is undoubtedly valuable in areas with pulsed emissions or discontinuous EMF sources. Moreover, it establishes a principled methodology to incorporate measurement bias and prior information in an optimal way from a Bayesian point of view. In addition, 


\section{UNCERTAINTY ANALYSIS IN ELECTROMAGNETIC FIELD MEASUREMENTS}

the presented techniques are extensible to other types of measurement and multivariate input or output quantities.

The developed framework entails several advantages concerning conventional uncertainty analysis techniques: (1) it utilises a non-parametric representation of EMF measurements to capture their dynamic nature, which is hindered by conventional uncertainty analyses; (2) it furnishes a systematic approach to incorporate prior information provided by historical data and previous knowledge, which is accomplished by means of importance sampling; (3) it enables the use of flexible prior densities such as kernel mixtures estimated by EM algorithm, which exhibit an outstanding performance in comparison with no informative priors; (4) it enhances the effectiveness of traditional methods specially for small sample sizes, which is often the case in the appraisal of exposure to EMFs.

The proposed adaptive techniques demonstrate a remarkable improvement in uncertainty analysis corroborated by actual broadband measurements of the E-field strength. Under the proposed framework, it is observed a significant uncertainty reduction that may play a crucial role in many related branches of science such as electromagnetic compatibility testing and prospective epidemiological studies.

\section{FUNDING}

This work was partially supported by the Regional Ministry of Health from Castilla y León (Spain); the Regional Ministry of Education from Castilla y León (Spain) and the European Social Fund.

\section{REFERENCES}

1. Ahlbom, I. C., Cardis, E., Green, A., Linet, M., Savitz, D. and Swerdlow, A. Review of the epidemiologic literature on EMF and health. Environ. Health Perspect. 109, 911-933 (2001).

2. Alonso, A., Bahillo, A., de la Rosa, R., Carrera, A., Durán, R. J. and Fernández, P. Measurement procedure to assess exposure to extremely low-frequency fields: a primary school case study. Radiat. Prot. Dosim. 151, 426-436 (2012).

3. Malagoli, C. et al. Maternal exposure to magnetic fields from high-voltage power lines and the risk of birth defects. Bioelectromagnetics 33, 405-409 (2012).

4. Nicolaou, C. P., Papadakis, A. P., Razis, P. A. and Kyriacou, G. A. Experimental measurement, analysis and prediction of electric and magnetic fields in open type air substations. Electric Power Syst. Res. 90, 42-54 (2012).

5. Repacholi, M. H. Low-level exposure to radio-frequency electromagnetic fields: health effects and research needs. Bioelectromagnetics 19, 1-19 (1998).

6. Kwon, M. S. and Hämäläinen, H. Effects of mobile phone electromagnetic fields: critical evaluation of behavioral and neurophysiological studies. Bioelectromagnetics 32, 253-272 (2011).

7. EU Council. Recommendation of 12 July 1999 on the limitation of exposure of the general public to electromagnetic fields (0 Hz to $300 \mathrm{GHz})(1999 / 519 / \mathrm{EC})$ (1999).

8. EU Council. Directive 2013/35/EU of the European Parliament and of the Council of 26 June 2013 (2013).

9. International Commission on Non-Ionizing Radiation Protection. Guidelines for limiting exposure to timevarying electric, magnetic, and electromagnetic fields (up to $300 \mathrm{GHz}$ ) (1998).

10. International Commission on Non-Ionizing Radiation Protection. Guidelines for limiting exposure to timevarying electric and magnetic fields $(1 \mathrm{~Hz}$ to $100 \mathrm{kHz}$ ) (2010).

11. IEEE recommended practice for measurements and computations of radio frequency electromagnetic fields with respect to human exposure to such fields, $100 \mathrm{kHz}-$ $300 \mathrm{GHz}$. IEEE Std C95.3-2002 (Revision of IEEE Std C95.3-1991) (2002).

12. European Committee for Electrotechnical Standardization. Human exposure to electromagnetic fields. High frequency $(10 \mathrm{kHz}$ to $300 \mathrm{GHz})$. CENELEC ENV 50166-2 (1995).

13. Lindley, D. V. Understanding Uncertainty. John Wiley \& Sons (2006).

14. Chadwick, P. Assessment uncertainties relating to electromagnetic fields (EMF) measurement and computation. Technical report. Health and Safety Executive (2008).

15. Joint Committee for Guides in Metrology. Evaluation of measurement data-Guide to the Expression of Uncertainty in Measurement (2008).

16. Alexandrov, Y. I. Uncertainty of measurement. Twenty years afterwards. Fresenius J. Anal. Chem. 370, 690-693 (2001).

17. Cox, M. G. and Harris, P. M. Measurement uncertainty and traceability. Meas. Sci. Technol. 17, 533-540 (2006).

18. Kacker, R. and Jones, A. On use of Bayesian statistics to make the Guide to the Expression of Uncertainty in Measurement consistent. Metrologia 5, 235-248 (2003).

19. Lira, I. and Grientschning, D. Equivalence of alternative Bayesian procedures for evaluating measurement uncertainty. Metrologia 47, 334-336 (2010).

20. Willink, R. and White, R. Disentangling classical and Bayesian approaches to uncertainty analysis. Technical report. Bureau International des Poids et Mesures (2012).

21. da Silva Hack, P. and ten Caten, C. S. Measurement uncertainty: literature review and research trends. IEEE Trans. Instrum. Meas. 61, 2116-2124 (2012).

22. Joint Committee for Guides in Metrology. Evaluation of measurement data-Supplement 1 to the 'Guide to the Expression of Uncertainty in Measurement'-propagation of distributions using a Monte Carlo method. Joint Committe for Guides in Metrology (2008).

23. Bernardo, J. M. and Smith, A. Bayesian Theory. John Wiley \& Sons (2000).

24. Mariscotti, A. Measurement procedures and uncertainty evaluation for electromagnetic radiated emissions from large-power electrical machinery. IEEE Trans. Instrum. Meas. 56, 2452-2463 (2007).

25. Stratakis, D. I., Miaoudakis, A. I., Xenos, T. D. and Zacharopoulos, V. G. Overall uncertainty estimation in multiple narrow-band in situ electromagnetic field mea- 


\section{J. PRIETO ET AL.}

surements. IEEE Trans. Instrum. Meas. 58, 2767-2779 (2009).

26. Stratakis, D. I., Miaoudakis, A. I., Zacharopoulos, V. G. and Xenos, T. D. On the spatial averaging of multiple narrowband electromagnetic field measurements: methods and uncertainty estimation. IEEE Trans. Instrum. Meas. 59, 1520-1536 (2010).

27. Vulevic, B. and Osmokrovic, P. Evaluation of uncertainty in the measurement of environmental electromagnetic fields. Radiat. Prot. Dosim. 141, 173-177 (2010).

28. Bolte, J. F. B., van der Zande, G. and Kamer, J. Calibration and uncertainties in personal exposure measurements of radiofrequency electromagnetic fields. Bioelectromagnetics 32, 652-663 (2011).

29. Bienkowski, P. and Trzaska, H. Electromagnetic Measurements in the Near Field. SciTech Publishing Inc (2012).

30. Ztoupis, I. N., Gonos, I. F. and Stathopulos, I. A. Uncertainty evaluation in the measurement of power frequency electric and magnetic fields from AC overhead power lines. Radiat. Prot. Dosim. 157, 11-21 (2013).

31. Carobbi, C. F. M. The GUM Supplement 1 and the uncertainty evaluations of EMC measurements. IEEEEMC Newsletter Spring 2010, 53-57 (2010).

32. Azpúrua, M., Tremola, C. and Páez, E. Comparison of the GUM and Monte Carlo methods for the uncertainty Estimation in electromagnetic compatibility testing. Prog. Electromagn Res. B 34, 125-144 (2011).

33. Robert, C. The Bayesian Choice. Springer Verlag (2007).

34. Turin, G. Communication through noisy, random-multipath channels. PhD thesis. Massachusetts Institute of Technology (1956).

35. International Telecommunication Union. Propagation data and prediction methods for the planning of shortrange outdoor radiocommunication systems and radio local area networks in the frequency range $300 \mathrm{MHz}$ to 100 $\mathrm{GHz}$. Recommendation ITU-R P.1411-7, International Telecommunication Union (2013).

36. Forbes, A. B. Measurement uncertainty and summarising Monte Carlo samples. In: XX IMEKO World Congress, Busan, Korea (2012).

37. Guimaraes, P. R., Carreteiro, J. and Pinheiro, S. Monte Carlo simulations applied to uncertainty in measurement. Theory Appl. Monte Carlo Simul. Tech. (2013).

38. Clifford, A. A. Multivariate Error Analysis: A Handbook of Error Propagation and Calculation in Many-parameter Systems. John Wiley \& Sons (1973).

39. Au, C. and Tam, J. Transforming variables using the Dirac generalized function. Am. Stat. 53, 270-272 (1999).

40. Parzen, E. On estimation of a probability density function and mode. Ann. Math. Stat. 33, 1065-1076 (1962).

41. Scott, D. W. Multivariate Density Estimation. John Wiley \& Sons (1992)

42. Prieto, J., Mazuelas, S., Bahillo, A., Fernández, P. and Lorenzo, R. M. Adaptive data fusion for wireless localization in harsh environments. IEEE Trans. Signal Process. 60, 1585-1596 (2012).

43. Chen, M. H. and Shao, Q. M. Monte Carlo estimation of Bayesian credible and HPD intervals. J. Comput. Graphical Stat. 8, 69-92 (1999).

44. Bernardo, J. M. Intrinsic credible regions: an objective Bayesian approach to interval estimation. TEST 14, 317-384 (2005).
45. Särkkä, S. Bayesian Filtering and Smoothing. Cambridge University Press (2013).

46. Rodríguez, B., Blas, J., Lorenzo, R. M., Fernández, P. and Abril, E. J. Statistical perturbations in personal exposure meters caused by the human body in dynamic outdoor environments. Bioelectromagnetics 32, 209-217 (2011).

47. Mahfouz, Z., Gati, A., Man-Fai, W., Wiart, J., Lautru, D. and Hanna, V. F. Statistical analysis of real radiofrequencies exposure in a realistic environment. In: Mediterranean Microwave Symposium (MMS), Guzelyurt, Turkey (2010).

48. McLachlan, G. and Peel, D. Finite Mixture Models. John Wiley \& Sons (2000).

49. Hashemi, H. The indoor radio propagation channel. Proc. IEEE 81, 943-968 (1993).

50. Larchevêque, E., Dale, C., Man-Faï, Wong. and Wiart, J. Analysis of electric field averaging for in situ radiofrequency exposure assessment. IEEE Trans. Vehicular Technol. 54, 1245-1250 (2005).

51. Prieto, J., Bahillo, A., Mazuelas, S., Lorenzo, R. M., Fernández, P. and Abril, E. J. Characterization and mitigation or range estimation errors for an RTT-based IEEE 802.11 indoor location system. Prog. Electromagn. Res. B 15, 217-244 (2009).

52. Freeden, J. V. EMR-20/-30 EMR-200/-300 operating manual. Technical report. Narda Safety Test Solutions $\mathrm{GmbH}$ (2001).

53. Bitzer, R. and Keller, H. Improved technique for simplifying standard-compliant tests of non-ionizing electromagnetic radiation. In: NIR 99 Annual Conference (1999).

54. IEEE standard for calibration of electromagnetic field sensors and probes, excluding antennas, from $9 \mathrm{kHz}$ to 40 GHz. IEEE Std 1309-2005 (Revision of IEEE Std 13091996), Insittute of Electrical and Electronics Engineers (2005).

\section{APPENDIX A. ALGORITHM FOR BAYESIAN UNCERTAINTY ANALYSIS IN EMF MEASUREMENTS}

Algorithm 1 Bayesian uncertainty analysis in EMF measurements.

1. Initialisation:

2. Draw $M$ samples $\left\{\tilde{y}_{m}\right\}_{m \in 1 \ldots ., M}$ from the importance density $\pi(y)$.

3. UPDATE:

4. For $m=1, \ldots, M$ do

5. (i) Weighting:

$$
\omega_{m} \leftarrow \frac{f\left(\tilde{y}_{m}\right) f\left(\mathbf{z} \mid \tilde{y}_{m}\right)}{\pi\left(\tilde{y}_{m}\right)} .
$$

\section{End for}

7. For $m=1, \ldots, M$ do

8. (ii) Normalisation:

$$
\tilde{\omega}_{m} \leftarrow \frac{\omega_{m}}{\sum_{m=1}^{M} \omega_{m}} .
$$




\section{End for}

10. (iii) ResAmPLING:

11. Draw $M$ samples $\left\{y_{m}\right\}_{m \in 1, \ldots, M}$ from the discrete distribution,

$$
f(y \mid \mathbf{z}) \approx \sum_{m=1}^{M} \tilde{\omega}_{m} \delta\left(y-\tilde{y}_{m}\right)
$$

12. SumMARISING:

13. Approximate the posterior expectation,

$$
A \leftarrow \sum_{m=1}^{M} \tilde{\omega}_{m} \tilde{y}_{m} \quad \text { or } \quad A \leftarrow \frac{1}{M} \sum_{m=1}^{M} y_{m} .
$$

14. Approximate the posterior density,

$$
\begin{aligned}
& B \leftarrow \frac{1}{h} \sum_{m=1}^{M} \tilde{\omega}_{m} K\left(\frac{y-\tilde{y}_{m}}{h}\right), \\
& \text { or } \\
& B \leftarrow \frac{1}{M h} \sum_{m=1}^{M} K\left(\frac{y-y_{m}}{h}\right) .
\end{aligned}
$$

15. Sort the set $\left\{y_{m}\right\}_{m \in 1, \ldots, M}$ and find,

$$
C \leftarrow \underset{r \in[1, M-q]}{\arg \min }\left\{y_{(r+q)}-y_{r}\right\},
$$

where $q=\lfloor p M+1 / 2\rfloor$ is the integer part of $(p M+1 / 2)$.

16. Compute the shortest $100 p \%$ credible interval (c.i.),

$$
[D, E] \leftarrow\left[y_{C}, y_{(C+q)}\right] .
$$

\section{Return}

$\mathbb{E}\{y \mid \mathbf{z}\} \leftarrow A ; \quad f(y \mid \mathbf{z}) \leftarrow B ; \quad$ c.i. $\leftarrow[D, E]$.

APPENDIX B. ADAPTIVE LIKELIHOOD FUNCTION FROM EMF STRENGTH MEASUREMENTS

Given a set of $S$ i.i.d. measurements, $\mathbf{z}=\left\{z_{i}\right\}_{i \in 1, \ldots, S}$, related to the EMF strength, $y$, and the systematic effects, $b$, by a model $\mathbb{E}\{z\}=y+b$, the distribution of a specific measurement, $z_{j}$, given $y$ and $b$, can be approximated by,

$$
f\left(z_{j} \mid y, b\right)=\frac{1}{S h} \sum_{i=1}^{S} K\left(\frac{\left(z_{j}-z_{i}+\mathbb{E}\{z\}\right)-(y+b)}{h}\right),
$$

where $K(\cdot)$ and $h$ are a kernel function and its bandwidth $^{(42)}$. By selecting a standard Gaussian kernel, it follows that $\mathbb{E}\{z\}=\overline{\mathbf{z}}$, where $\overline{\mathbf{z}}$ is the sample mean of the set $\mathbf{z}=\left\{z_{i}\right\}_{i \in 1, \ldots, S}$. Therefore, the distribution of a specific measurement, $z_{j}$, given $y$ and $b$, turns into

$$
f\left(z_{j} \mid y, b\right)=\frac{1}{\sqrt{2 \pi} S h} \sum_{i=1}^{S} \exp \left(-\frac{\left(z_{j}-z_{i}+\overline{\mathbf{z}}-y-b\right)^{2}}{2 h^{2}}\right) .
$$

Then, the distribution of the set of measurements given $y$ and $b$ is,

$$
f(\mathbf{z} \mid y, b)=\prod_{j=1}^{S} f\left(z_{j} \mid y, b\right)
$$

And the likelihood function of the field strength can be approximated by marginalising out the bias,

$$
\begin{aligned}
f(\mathbf{z} \mid y) & =\int f(\mathbf{z} \mid y, b) f(b) \mathrm{d} b \\
& =\mathbb{E}_{b}\{f(\mathbf{z} \mid y, b)\},
\end{aligned}
$$

where $\mathbb{E}_{b}\{\cdot\}$ stands for the expectation with respect to the bias, $b$.

APPENDIX C. ALGORITHM FOR THE EVALUATION OF THE ADAPTIVE LIKELIHOOD

Algorithm 2 Function to evaluate the sample $\tilde{y}_{m}$ in the likelihood given by Equation (12).

1: Function LIKELIHOOD_UPDATE $\left(\tilde{y}_{m}, \mathbf{z}, f(b)\right)$

2: INITIALIZATION:

3: Draw $N$ samples $\left\{b_{n}\right\}_{n \in 1, \ldots . N}$ from the known bias PDF $f(b)$.

4: Bias evaluation:

5: For $n=1, \ldots, N$ do

6: For $j=1, \ldots, S$ do

7: Evaluate $f\left(z_{j} \mid y, b\right)$,

$$
A_{j} \leftarrow \frac{1}{\sqrt{2 \pi} S h} \cdot \sum_{i=1}^{S} \exp \left(-\frac{\left(z_{j}-z_{i}+\overline{\mathbf{z}}-\tilde{y}_{m}-b_{n}\right)^{2}}{2 h^{2}}\right) .
$$

8: End for

9: Evaluate $f(\mathbf{z} \mid y, b)$,

$$
B_{n} \leftarrow \prod_{j=1}^{S} A_{j}
$$

\section{0: End for}

11: Marginalization:

12: Approximate the likelihood of $\tilde{y}_{m}$,

$$
C \leftarrow \frac{1}{N} \sum_{n=1}^{N} B_{n} .
$$

13: Return $f\left(\mathbf{z} \mid \tilde{y}_{m}\right) \leftarrow C$.

14: End function 